\title{
Ground-state stability and criticality of two-electron atoms with screened Coulomb potentials using the B-splines basis set
}

\author{
Pablo Serra* \\ Department of Chemistry, Purdue University, \\ West Lafayette, Indiana 47907, USA and \\ Facultad de Matemática, Astronomía y Física, \\ Universidad Nacional de Córdoba, and IFEG-CONICET, \\ Ciudad Universitaria, X5000HUA, Córdoba, Argentina \\ Sabre Kaist \\ Department of Chemistry, Purdue University, \\ West Lafayette, Indiana 4790\%, USA \\ Department of Physics, Purdue University, \\ West Lafayette, Indiana 4790\%, USA and \\ Qatar Environment and Energy Research Institute, Qatar Foundation, Doha, Qatar
}




\begin{abstract}
We applied the finite-size scaling method using the B-splines basis set to construct the stability diagram for two-electron atoms with a screened Coulomb potential. The results of this method for two electron atoms are very accurate in comparison with previous calculations based on Gaussian, Hylleraas, and finite-element basis sets. The stability diagram for the screened two-electron atoms shows three distinct regions: a two-electron region, a one-electron region, and a zero-electron region, which correspond to stable, ionized and double ionized atoms. In previous studies, it was difficult to extend the finite size scaling calculations to large molecules and extended systems because of the computational cost and the lack of a simple way to increase the number of Gaussian basis elements in a systematic way. Motivated by recent studies showing how one can use B-splines to solve Hartree-Fock and Kohn-Sham equations, this combined finite size scaling using the B-splines basis set, might provide an effective systematic way to treat criticality of large molecules and extended systems. As benchmark calculations, the two-electron systems show the feasibility of this combined approach and provide an accurate reference for comparison.
\end{abstract}

* serra@famaf.unc.edu.ar

$\dagger$ kais@purdue.edu 


\section{INTRODUCTION}

Weakly bound systems represent an interesting field of research in atomic and molecular physics. The behavior of systems near a binding threshold is important in the study of ionization of atoms and molecules, molecule dissociation, and scattering collisions. Since the pioneering works of Bethe [1] and Hylleraas [2] confirming the existence of the negative hydrogen ion, $H^{-}$, the study of the stability of the ground state of atomic and molecular negative anions becomes an active field of research. New phenomena appear when the Coulomb interaction is screened and the long-range electrostatic interactions turn to short-range potentials. A simple model to describe the effect of the screening in the Coulomb potential is the Yukawa potential, where an exponential decay is introduced, $1 / r \rightarrow \exp (-r / D) / r$, where $D$ is a positive constant. The Yukawa potential has been used in many branches of physics, for example to describe interactions in dusty plasmas where charged dust particles are surrounded by plasma [3], liquid metals [4], charged colloidal particles [5]. Two-electron systems interacting via Yukawa potentials were the subject of recent research, studying the bound states using the Hylleraas basis set [6-10] and B-spline expansions [11]. Also, scattering processes were recently presented [12] using Yukawa potentials.

To examine near threshold behavior, the Finite Size Scaling (FSS) approach is needed in order to extrapolate results obtained from finite systems to the complete basis set limit. FSS is not only a formal way to understand the asymptotic behavior of a system when the size tends to infinity, but a theory that also gives us numerical methods capable of obtaining accurate results for infinite systems by studying the corresponding small systems 13 24]. Applications include expansion in Slater-type basis functions [22], Gaussian-type basis functions [25] and recently, finite elements [26].

Here, we combine FSS with the B-splines expansion to calculate the stability diagram for two-electron atoms with a screened Coulomb potential. The B-spline functions $B_{i}(r), i=$ $1, n_{s}$ form a basis for piecewise polynomial functions that are polynomials of degree $\left(k_{s}-1\right)$ in each interval and whose derivatives up to order $\left(k_{s}-2\right)$ are continuous at the interior knots, have been increasingly used in atomic and molecular physics [27 31]. Our results show the B-splines functions are very efficient in performing FSS to calculate the critical parameters and the stability diagram.

The paper is organized as follows. In Section II we present FSS with a B-splines basis 
followed by the two-electron atom, as a benchmark calculation in Section [II]. In Section [I] we present our main results for the screened two-electron atoms. Finally, in Section $\nabla$ we discuss our results and conclusions.

\section{FINITE SIZE SCALING (FSS) WITH B-SPLINES}

Here, we briefly introduce the finite size scaling (for more details, see Ref. [22]) and how to perform calculations using B-splines. The finite size scaling method is a systematic way to extract the critical behavior of an infinite system from analysis on finite systems [22]. It is efficient and accurate for the calculation of critical parameters for few-body Schrödinger equation.

In our study, we have Hamiltonian of the following form:

$$
\mathcal{H}=\mathcal{H}_{0}+V_{\lambda}
$$

where $\mathcal{H}_{0}$ is $\lambda$-independent and $V_{\lambda}$ is the $\lambda$-dependent term. We are interested in the study of how the different properties of the system change when the value of $\lambda$ varies. A critical point, $\lambda_{c}$, will be defined as a point for which a bound state becomes absorbed or degenerate with a continuum. We also define a critical exponent $\alpha$ by the asymptotic behavior of the ionization energy $E(\lambda)-\mathcal{E}_{t h} \sim\left(\lambda-\lambda_{c}\right)^{\alpha}$, where we assume that the threshold energy, $\mathcal{E}_{t h}$, does not depend on $\lambda$. In the first example, the He-like atoms, we have only one parameter, $\lambda$ while for the second case, the screened two electron atoms, we have two parameters, $\lambda_{1}$ and $\lambda_{2}$. To perform the finite size scaling calculations, we expand the exact wave function in a finite basis set and truncate this expansion at some order $N$. The finite size corresponds to the number of elements in a complete basis set used to expand the exact eigenfunction of a given Hamiltonian. The ground-state eigenfunction has the following expansion: $\Psi_{\lambda}=\sum_{n} a_{n}(\lambda) \phi_{n}$,

where $\mathrm{n}$ is the set of quantum numbers. We have to truncate the series at order $\mathrm{N}$, and the expectation value of any general operator $\mathcal{O}$ at order $\mathrm{N}$ is given by:

$$
\langle\mathcal{O}\rangle^{N}=\sum_{n, m}^{N} a_{n}^{(N)} a_{m}^{(N)} \mathcal{O}_{n, m},
$$

where $\mathcal{O}_{n, m}$ are the matrix elements of $\mathcal{O}$ in the basis set $\left\{\phi_{n}\right\}$.

In this study we used the B-splines basis, the normalized one-electron orbitals are given 
by

$$
\phi_{n}(r)=C_{n} \frac{B_{n+1}^{(k)}(r)}{r} ; n=1, \ldots
$$

where $B_{n+1}^{(k)}(r)$ is a B-splines polynomial of order $k$. The numerical results are obtained by defining a cutoff radius $R$, and then the interval $[0, R]$ is divided into $I$ equal subintervals. B-spline polynomials [31] (for a review of applications of B-splines polynomials in atomic and molecular physics, see ref. [33]) are piecewise polynomials defined by a sequence of knots $t_{1}=0 \leq t_{2} \leq \cdots \leq t_{2 k+I-1}=R$ and the recurrence relations

$$
\begin{gathered}
B_{i, 1}(r)=\left\{\begin{array}{l}
1 \text { if } t_{i} \leq r<t_{i+1} \\
0 \text { otherwise, }
\end{array} .\right. \\
B_{i, k}(r)=\frac{r-t_{i}}{t_{i+k-1}-t_{i}} B_{i, k-1}(r)+\frac{t_{i+k}-r}{t_{i+k}-t_{i+1}} B_{i, k-1}(r)(k>1) .
\end{gathered}
$$

In this work, we use the standard choice for the knots in atomic physics [33] $t_{1}=\cdots=t_{k}=0$ and $t_{k+I}=\cdots=t_{2 k+I-1}=R$. Because we are interested in FSS, we choose an equidistant distribution of inside knots. The constant $C_{n}$ in Eq.(3) is a normalization constant obtained from the condition $\langle n \mid n\rangle=1$,

$$
C_{n}=\frac{1}{\left[\int_{0}^{R_{0}}\left(B_{n+1}^{(k)}(r)\right)^{2} d r\right]^{1 / 2}} .
$$

Because $B_{1}(0) \neq 0$ and $B_{I+k-1}(R) \neq 0$, we have $N=I+k-3$ orbitals corresponding to $B_{2}, \ldots, B_{I+k-2}$. In all the calculations we used the value $k=5$, and, we do not write the index $k$ in the eigenvalues and coefficients.

To obtain the numerical values of the critical parameters $\left(\lambda_{c}, \alpha\right)$ for the energy, we define for any given operator $\mathcal{O}$ the function

$$
\triangle_{\mathcal{O}}\left(\lambda ; N, N^{\prime}\right)=\frac{\ln \left(\left\langle\mathcal{O}_{\lambda}^{N}\right\rangle /\langle\mathcal{O}\rangle_{\lambda}^{N^{\prime}}\right)}{\ln \left(N^{\prime} / N\right)},
$$

If we take the operator $\mathcal{O}$ to be $H-\mathcal{E}_{t h}$, and $\partial H / \partial \lambda$, we can obtain the critical parameters from the following function [22]

$$
\Gamma_{\alpha}\left(\lambda, N, N^{\prime}\right)=\frac{\triangle_{H}\left(\lambda ; N, N^{\prime}\right)}{\triangle_{H}\left(\lambda ; N, N^{\prime}\right)-\triangle_{\frac{\partial H}{\partial \lambda}}\left(\lambda ; N, N^{\prime}\right)},
$$


which at the critical point is independent of $N$ and $N^{\prime}$ and takes the value of $\alpha$. Namely, for $\lambda=\lambda_{c}$ and any values of $N$ and $N^{\prime}$ we have

$$
\Gamma_{\alpha}\left(\lambda_{c}, N, N^{\prime}\right)=\alpha
$$

Because our results are asymptotic for large values of $N$, we obtain a sequence of pseudocritical parameters $\left(\lambda_{N}, \alpha_{N}\right)$ that converge to $\left(\lambda_{c}, \alpha\right)$ for $N \rightarrow \infty$.

\section{HELIUM-LIKE ATOMS}

As a benchmark for FSS using B-splines we calculate the critical parameters of the twoelectron atom with standard Coulomb potential. In this case, after a scaling with the nuclear charge, the system has a unique parameter $\lambda=1 / Z$

$$
H=-\frac{1}{2} \nabla_{\mathbf{r}_{1}}^{2}-\frac{1}{2} \nabla_{\mathbf{r}_{2}}^{2}-\frac{1}{r_{1}}-\frac{1}{r_{2}}+\lambda \frac{1}{\left|\mathbf{r}_{2}-\mathbf{r}_{1}\right|} .
$$

The ground-state energy $E_{0}\left(\lambda_{1}, \lambda_{2}\right)$ and its corresponding eigenvector $\left|\psi_{0}(1,2)\right\rangle$ will be calculated within the variational approximation

$$
\left|\psi_{0}(1,2)\right\rangle \simeq\left|\Psi_{0}(1,2)\right\rangle=\sum_{i=1}^{M} c_{i}^{(j)}\left|\Phi_{i}\right\rangle, \quad c_{i}^{(j)}=\left(\mathbf{c}^{(j)}\right)_{i} ; j=1, \cdots, M .
$$

where the $\left|\Phi_{i}\right\rangle$ must be chosen adequately and $M$ is the basis set size.

Since we are interested in the behavior of the system near the ground-state ionization threshold, we choose as basis set s-wave singlets given by

$$
\left|\Phi_{i}\right\rangle \equiv\left|n_{1}, n_{2} ; l\right\rangle=\left(\phi_{n_{1}}\left(r_{1}\right) \phi_{n_{2}}\left(r_{2}\right)\right)_{s} \mathcal{Y}_{0,0}^{l}\left(\Omega_{1}, \Omega_{2}\right) \chi_{s}
$$

where $n_{2} \leq n_{1} \leq N$. Also, we introduce a cutoff value $l_{\max }$ for the angular momentum $l \leq l_{\max }$, denote $\chi_{s}$ as the singlet spinor, and the $\mathcal{Y}_{0,0}^{l}\left(\Omega_{1}, \Omega_{2}\right)$ are given by

$$
\mathcal{Y}_{0,0}^{l}\left(\Omega_{1}, \Omega_{2}\right)=\frac{(-1)^{l}}{\sqrt{2 l+1}} \sum_{m=-l}^{l}(-1)^{m} Y_{l m}\left(\Omega_{1}\right) Y_{l-m}\left(\Omega_{2}\right)
$$

i.e. they are eigenfunctions of the total angular momentum with zero eigenvalue, and the $Y_{l m}$ are the spherical harmonics. Note also that $\mathcal{Y}_{0,0}^{l}$ is a real function since it is symmetric in the particle index. The radial term $\left(\phi_{n_{1}}\left(r_{1}\right) \phi_{n_{2}}\left(r_{2}\right)\right)_{s}$ has the appropriate symmetry for a singlet state, 


$$
\left(\phi_{n_{1}}\left(r_{1}\right) \phi_{n_{2}}\left(r_{2}\right)\right)_{s}=\phi_{n_{1}}\left(r_{1}\right) \phi_{n_{2}}\left(r_{2}\right)+\phi_{n_{1}}\left(r_{2}\right) \phi_{n_{2}}\left(r_{1}\right)
$$

In general, the size $M$ of a basis set defined for Eqs.(11,14) is $M=N(N+1)\left(l_{\max }+1\right) / 2$. For the radial orbitals we used normalized B-splines polynomial of order $k$

$$
\phi_{n}(r)=C_{n} \frac{B_{n+1}^{(k)}(r)}{r} ; n=1, \ldots, N=k+I-3 .
$$

The calculations in this sections were done with $k=5, R=30$, and $l_{\max }=3$.

In order to calculate the Hamiltonian matrix elements, we expand the electronic Coulomb interaction in spherical harmonics

$$
\frac{1}{\left|\mathbf{r}_{2}-\mathbf{r}_{1}\right|}=\sum_{l=0}^{\infty} \frac{4 \pi}{2 l+1} \frac{r_{<}^{l}}{r_{>}^{l+1}} \sum_{m=-l}^{l} Y_{l, m}^{*}\left(\Omega_{1}\right) Y_{l, m}\left(\Omega_{2}\right),
$$

Because the cutoff $l_{\max }$, the matrix elements of this expansion are nonzero only for $l \leq$ $2 l_{\max }$

In our previous studies, the critical behavior of the two-electron atom was obtained by using FSS approach with Hylleraas [34] and Gaussian basis sets [35]. The FSS was performed with a finite small basis-set and then increased the number of basis functions $N$ in a systematic way. The B-splines basis-set, in this sense is different. When $N$ is changed, we are not adding new functions, but the complete basis-set is changed in a way which is similar to the finite-element method [36].

Figure 1, shows the results for the plot $\Gamma_{N}$ as $\lambda$ varies for different values of $N$. Successive curves cross at pseudocritical points. In Figures (2) and (3), we observed the behavior of the pseudocritical parameters, $\lambda_{N}$ and $\alpha_{N}$ as a function of $1 / N$. The two curves converged to the exact values, in complete agreement with our previous [17, 22, 25] and recent results [37]. The numerical values are shown in table I. These accurate results indicate that FSS can be combined with B-spline basis to obtain critical parameters for the few-body Schrödinger equation.

\section{THE SCREENED TWO-ELECTRON ATOM}

The Hamiltonian, in atomic units, takes the form,

$$
H=-\frac{1}{2} \nabla_{\mathbf{r}_{1}}^{2}-\frac{1}{2} \nabla_{\mathbf{r}_{2}}^{2}-\frac{Z e^{-r_{1} / D}}{r_{1}}-\frac{Z e^{-r_{2} / D}}{r_{2}}+\frac{e^{-\left|\mathbf{r}_{2}-\mathbf{r}_{1}\right| / D}}{\left|\mathbf{r}_{2}-\mathbf{r}_{1}\right|},
$$




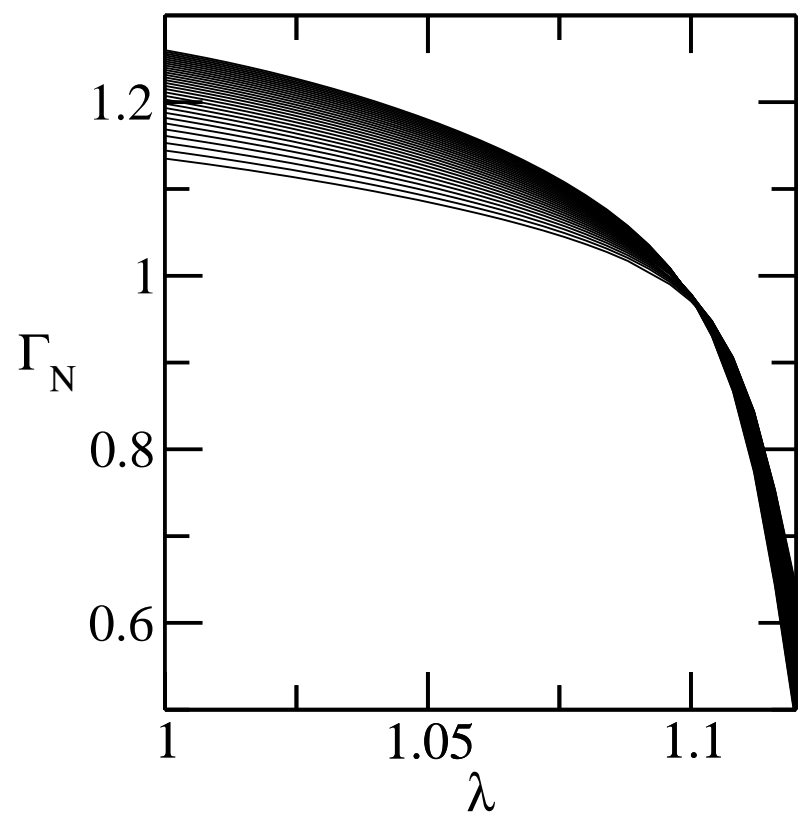

FIG. 1. (color-online) $\Gamma_{N}$ vs. $\lambda$ for two-electron atoms, for $N=20, \cdots, 50$.

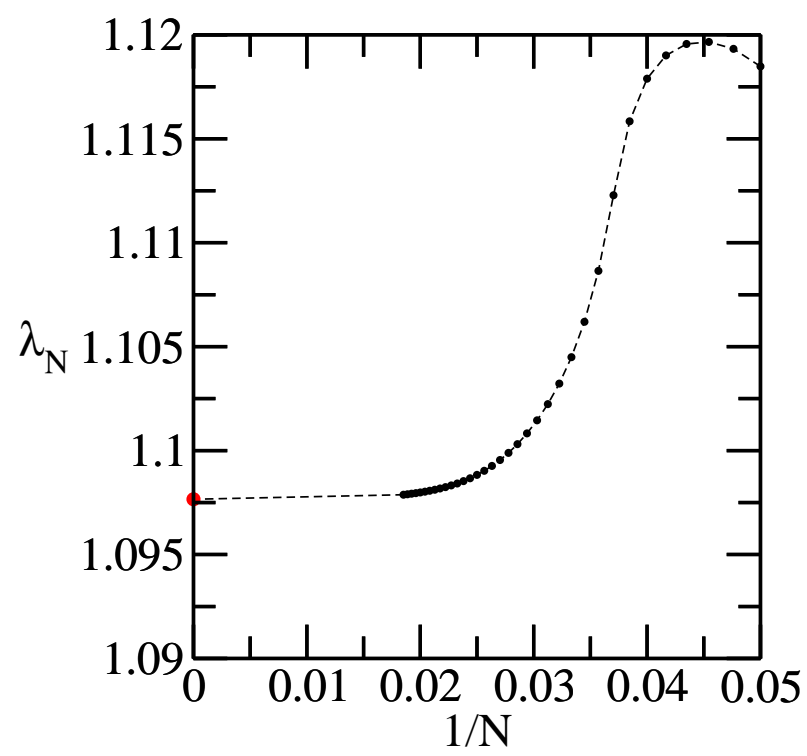

FIG. 2 . $\lambda_{N}$ vs. $1 / N$ for $N=20, \cdots, 55$ for the two-electron atom. The red point is the value of $\lambda_{c}$ from ref.[34] .

where $Z$ is the nuclear charge and $D>0$ the Debye screening length. The Hamiltonian takes a form more convenient to our purposes after scaling with $D, r \rightarrow r / D$ and $H \rightarrow D^{2} H$ 


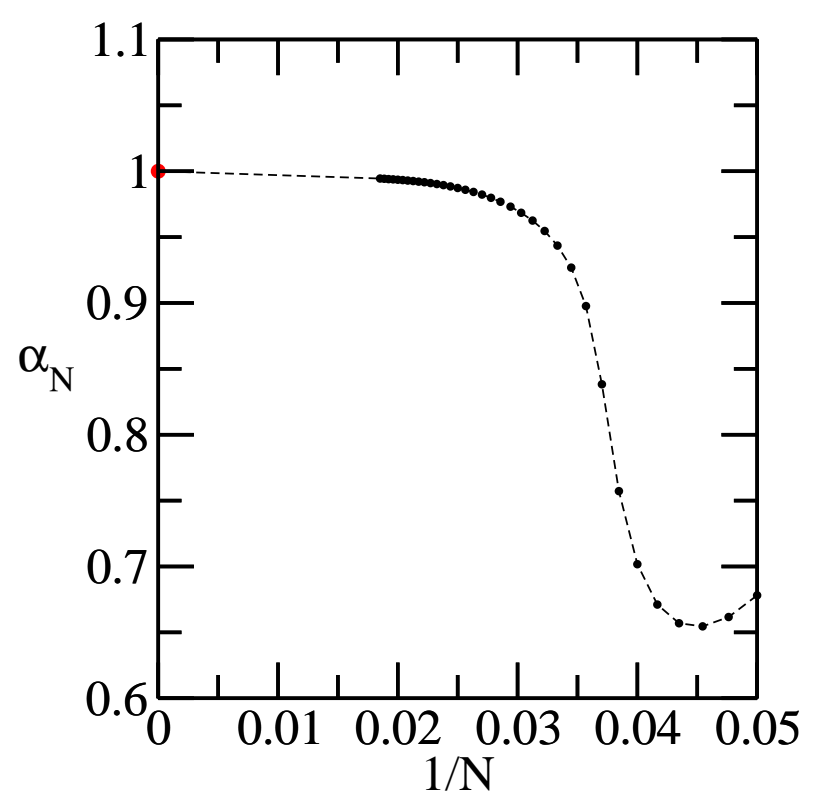

FIG. 3. (color-online) Critical exponent $\alpha_{N}$ vs. $1 / N$ for $N=20, \cdots, 55$ for the two-electron atom. The red point is the exact value $\alpha=1$.

\begin{tabular}{|c|c|c|c|c|}
\hline \hline & This work & FSS (Hylleraas) [22] & Ref [37] & exact \\
\hline \hline$\lambda_{c}$ & 1.09776 & 1.0976 & 1.09788 & - \\
\hline$\alpha$ & 0.9947 & 1.04 & - & 1 \\
\hline \hline
\end{tabular}

TABLE I. Comparison of $\lambda_{c}$ and $\alpha$ for the ground-state energy of the two-electron atom.

$$
H=-\frac{1}{2} \nabla_{\mathbf{r}_{1}}^{2}-\frac{1}{2} \nabla_{\mathbf{r}_{2}}^{2}-\lambda_{1}\left(\frac{e^{-r_{1}}}{r_{1}}+\frac{e^{-r_{2}}}{r_{2}}\right)+\lambda_{2} \frac{e^{-\left|\mathbf{r}_{2}-\mathbf{r}_{1}\right|}}{\left|\mathbf{r}_{2}-\mathbf{r}_{1}\right|},
$$

where $\lambda_{1}=Z D$, and $\lambda_{2}=D$. The numerical results are obtained using the same basis set as the Coulomb case described in section III, except the value of the cutoff radius, that for the Yukawa potential we set as $R=20$.

For the Yukawa potential we use the Gegenbauer's expansion in spherical harmonics

$$
\frac{e^{-\left|\mathbf{r}_{2}-\mathbf{r}_{1}\right|}}{\left|\mathbf{r}_{2}-\mathbf{r}_{1}\right|}=\sum_{l=0}^{\infty} 4 \pi \frac{I_{l+1 / 2}\left(r_{<}\right)}{\sqrt{r_{<}}} \frac{K_{l+1 / 2}\left(r_{>}\right)}{\sqrt{r_{>}}} \sum_{m=-l}^{l} Y_{l, m}^{*}\left(\Omega_{1}\right) Y_{l, m}\left(\Omega_{2}\right),
$$

where $I_{l+1 / 2}$ and $K_{l+1 / 2}$ are the modified Bessel functions of the first and second kind, respectively [38].

Since $\mathcal{E}_{\text {th }}$ does not depend on $\lambda_{2}$, we calculate the scaling function $\Gamma_{N}$ for given values 
of $\lambda_{1}$ as a function of $\lambda_{2}$. Figure 4, show the results for the plot $\Gamma_{N}\left(\lambda_{1}=1.5 ; \lambda_{2}\right)$ as $\lambda_{2}$ varies for different values if $N$. All the curves cross very close to the critical point. In Figure 5 we present the phase diagram for the screened two-electron atoms with three distinct phases: two-electron phase $\left(2 e^{-}\right)$, one-electron phase $\left(1 e^{-}\right)$and zero-electron phase $\left(0 e^{-}\right)$, corresponding to stable, ionized and double ionized atoms.

The dotted line $\lambda_{1}=\lambda_{1}^{(c)} \simeq 0.84$ corresponds to the critical value of the one-electron Yukawa potential. Therefore, there are no bound states for $\lambda_{1} \leq \lambda_{1}^{(c)}$. For $\lambda_{1} \gtrsim \lambda_{1}^{(c)}$ the onebody bound state is extended, and the method is applicable until the size of the one-body state becomes of the order of the cutoff radius $R$. For the value $R=20$, we calculate the $1 e^{-}$stability line for $\lambda_{1} \geq 0.95$.

Reference [32] described the three different ground-state stability diagrams that a twoparameter Hamiltonian with short-range one-body potential could present. These cases are (see figure 1 of this reference) (a) no $2 e^{-}-0 e^{-}$line, (b) exists a finite $2 e^{-}-0 e^{-}$line for

$0 \leq \lambda_{2} \leq \lambda_{2}^{(m c)}$, (c) the $2 e^{-}-0 e^{-}$line is infinite. Also in this reference rigorous lower and upper bound for the $2 e^{-}-1 e^{-}$stability line are established. We calculate these bounds for the Hamiltonian Eq(18). The lower bound is shown in figure 55. For the Yukawa potential the upper bound diverges for $\lambda_{1} \rightarrow \lambda_{1}^{(c)}$, and then it is not useful in this case.

Even our results suggest that the ground-state stability diagram is of type (a). Large numerical instabilities could appear for $\lambda_{1} \rightarrow \lambda_{1}^{(c)}$, and then we can discard a type (c) diagram, but we can not discard a type (b) diagram with a small value of $\lambda_{2}^{(m c)}$.

We note that the $H^{-}$atom corresponds to the line $\lambda_{2}=\lambda_{1}$ and the He atom to the line $\lambda_{2}=\lambda_{1} / 2$. These lines are also indicated in figure 5. The critical screening values for $\mathrm{H}^{-}$ and He are $D_{H^{-}} \simeq 1.2969$ and $D_{H e} \simeq 0.4934$ respectively.

In Figure 6 we show how the critical exponent $\alpha^{N}$ vs $\lambda_{1}$ for $N=40$ for the screened two-electron atom converges to the exact value, $\alpha=1$ [32].

\section{SUMMARY AND CONCLUSIONS}

We have shown that the introduction of B-spline basis sets in FSS calculations is very powerful in obtaining critical parameters and stability diagrams for few-body systems.

This basis set presents very different characteristics than the standard basis sets previously used in FSS like Hylleraas or Slater-type basis sets. B-splines are non-zero only on a 


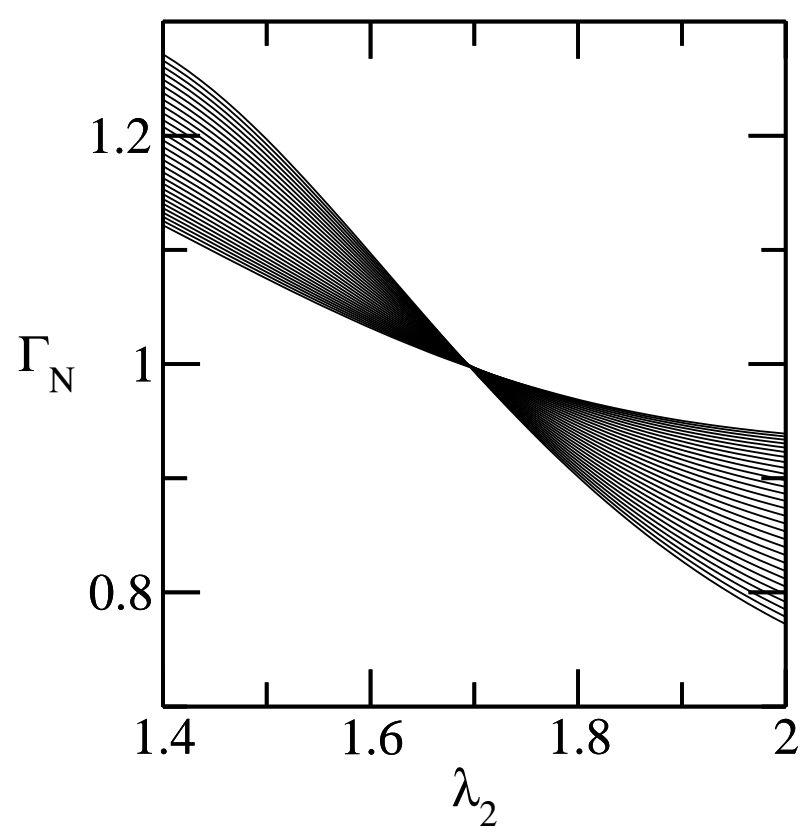

FIG. 4. (color-online) $\Gamma_{N}\left(\lambda_{1}=1.5 ; \lambda_{2}\right)$ vs. $\lambda_{2}$ for the screened two-electron atom, for $N=$ $20, \cdots, 50$.

small interval, and changing the FSS parameter N (number of basis functions) changes the complete basis set. In particular, we used this basis set together with FSS to calculate the critical parameter of the helium-like atom as a benchmark, finding very accurate results. We then applied the method to the important case of the ground-state stability diagram for a two-electron atom interacting via a screened Coulomb potential. Also in this case, FSS with a B-spline basis-set proves to be an excellent approach to obtain the critical behavior for this two-parameter Hamiltonian.

Our results show that the ground-state diagram of two-electron atoms interacting via Yukawa potentials does not present a $2 e^{-}-0 e^{-}$line. That is, the systems always undergoes a $2 e^{-}-1 e^{-}$transition before losing both electrons as the screening grows. Even the numerical results are not accurate enough to discard a small $2 e^{-}-0 e^{-}$line. We discard the existence of an infinite $2 e^{-}-0 e^{-}$line.

We have shown in previous works that FSS combined with different basis functions (Hylleraas, Gaussian, Slater) is a powerful method to obtain quantum critical parameters for few-body systems [22]. However, these basis sets are not useful to calculate critical parameter for large systems, or for quantum phase transitions in infinite systems. A possible 


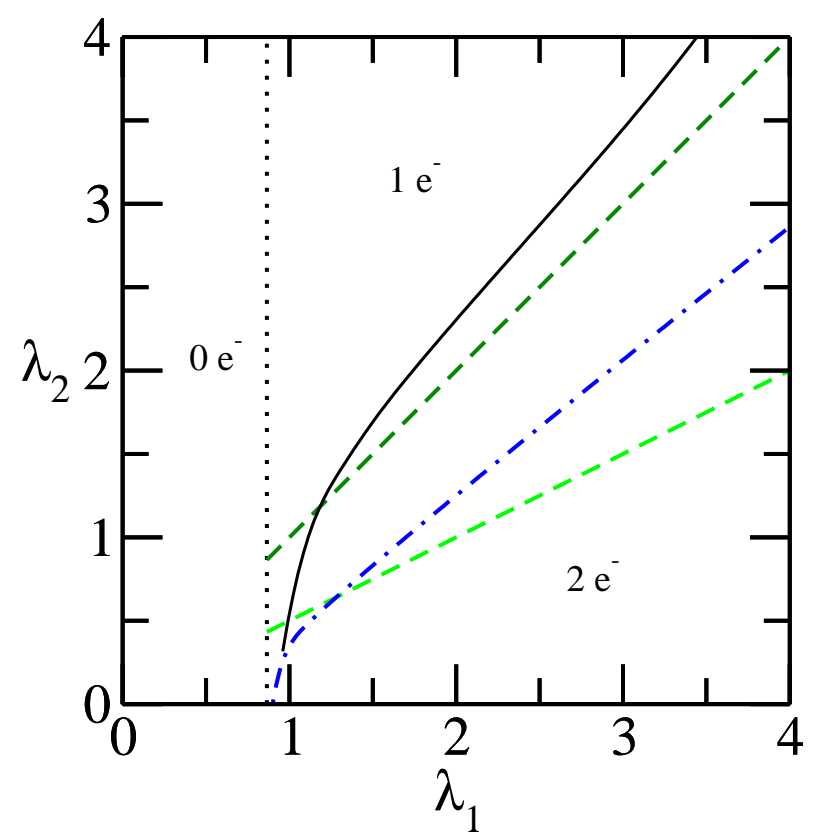

FIG. 5. (color-online) Ground-state stability diagram for the screened two-electron atom, the black line is the critical $2 e^{-}-1 e^{-}$line calculated with FSS with $N=40$, the dot line is the $1 e^{-}-0 e^{-}$critical line, the dashed blue line is the lower bound for the $2 e^{-}-1 e^{-}$line of ref. [32]. The dot-dashed lines correspond to the Helium (dark green) and Hydrogen (light green) atoms respectively.

way to apply FSS to study quantum phase transitions in materials is to combine FSS with Hatree-Fock or density functional approaches. In this direction, new efficient methods to solve the Hartree-Fock equations using B-splines expansions were recently established [39], and numerical codes are available [27]. As a benchmark system, we started with the two electron atoms. We show that indeed this can be done and obtained very accurate quantum critical parameters. Then we went to a more difficult case, two-electron atoms with screened Coulomb potentials. Getting all the stability and transition lines from two-electrons to oneelectron to zero-electrons is numerically difficult calculations. We have shown that FSS with B-spline basis functions can construct the full stability diagram. Our work is in progress to calculate critical parameters for large i molecular and extended systems by applying FSS with B-spline expansions of Hatree-Fock equations. 


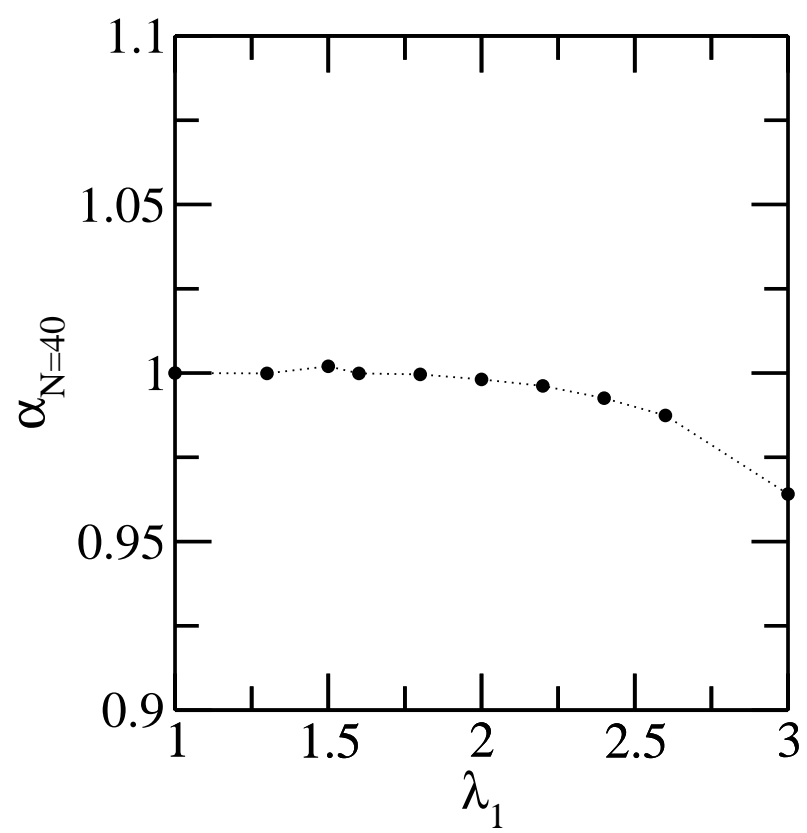

FIG. 6. (color-online) Critical exponent $\alpha_{N}$ vs $\lambda_{1}$ for $N=40$ for the screened two-electron atom. The exact value is $\alpha=1$.

\section{ACKNOWLEDGMENTS}

This work is supported by the NSF Centers for Chemical Innovation: Quantum Information and Computation for Chemistry, CHE-1037992. P. Serra would like to acknowledge the hospitality of Purdue University, where the work was done, and partial financial support of SECYT-UNC, CONICET, Min-CyT-Córdoba and the Programa Cuarto Centenario de la Universidad Nacional de Córdoba.

[1] H. Bethe, Z. Phys. 54, 347, (1929).

[2] E.A. Hylleraas, 1930 Z. Phys. 63, 739.

[3] D. Salzman, Atomic Physics in Hot Plasmas (Oxford University Press, Oxford, 1998), 1st ed.

[4] J. N. Herrera, P. T. Cummings, and H. Ruíz-Estrada, Mol. Phys. 96, 835 (1999).

[5] M. Heinen, P. Holmqvist, A. J. Banchio, and G. Nägele, J. Chem. Phys. 134, 044532 (2011).

[6] S. Kar, and Y. K. Ho, Int. J. Quan. Chem. 106, 814 (2006).

[7] F. Pont and P. Serra, Phys. Rev. A 79, 032508 (2009). 
[8] S. Kar, Y. K. Ho, and Z. Jiang, J. Phys. B 44165004 (2011).

[9] S. Sen, P. Mandal, and P. K. Mukherjee, Phys. Plasmas 19, 033501 (2012)

[10] Z. Jiang, S. Kar, and Y. K. Ho, Phys. Plasmas 19, 033301 (2012)

[11] Y.-C. Lin, C.-Y. Lin, and Y. K. Ho, Phys. Rev. A 85, 042516 (2012).

[12] M. C. Zammit, D. V. Fursa, I. Bray, and R. K. Janev, Phys. Rev. A 84, 052705 (2011).

[13] J.P. Neirotti, P. Serra, and S. Kais, Phys. Rev. Lett., 79, 3142 (1997).

[14] P. Serra, J.P. Neirotti, and S. Kais, Phys. Rev. Lett. 80, 5293 (1998).

[15] P. Serra and S. Kais, Phys. Rev. Letters, 77, 466 (1996).

[16] P. Serra, J.P. Neirotti, and S. Kais, Phys. Rev. A 57, R1481 (1998).

[17] P. Serra, J.P. Neirotti, and S. Kais, J. Phys. Chem. A 102, 9518 (1998).

[18] J.P. Neirotti, P. Serra, and S. Kais, J. Chem. Phys. 108, 2765 (1998).

[19] Q. Shi and S. Kais, Mol. Phys. 98, 1485 (2000).

[20] S. Kais and Q Shi, Phys. Rev. A 62, 60502 (2000).

[21] S. Kais, and P. Serra, Int. Rev. Phys. Chem. 19, 97 (2000).

[22] S. Kais, and P. Serra, Adv. Chem. Phys., 125, 1 (2003).

[23] P. Serra, and S. Kais, Chem. Phys. Lett. 372, 205-209 (2003).

[24] A. Ferron, P. Serra and S. Kais, J. Chem. Phys. 120, 8412-8419 (2004).

[25] W. Moy, P. Serra, and S. Kais, Mol. Phys. 106, 203 (2008).

[26] W. Moy, M. Carignano and S. Kais, J. Phys. Chem. 112, 5448-5452 (2008).

[27] C.Froese Fischer, Comp. Phys. Commun. 182, 1315 (2011).

[28] F. Martin, J. Phys. B, 32, R197 (1999)

[29] C.W. McCurdy, M. Baertschy, and T.N. Rescigno, J. Phys. B 37, R137 (2004).

[30] H. Bachau, E. Cormier, P. Decleva, J E Hansen and F. Martin, Rep. Prog. Phys. 64, 1815 (2001).

[31] C. de Boor, A Practical Guide to Splines (Springer, New York, 2001).

[32] F. Pont and P. Serra, J. Phys. A 41, 275303 (2008).

[33] H. Bachau, E. Cormier, P. Decleva, J. E. Hansen, and F. Martin, Rep. Prog. Phys. 64, 1815 (2001).

[34] J.P. Neirotti, P. Serra, and S. Kais, J. Chem. Phys. 108, 2765 (1998).

[35] W. Moy, S. Kais and P. Serra, Mol. Phys. 106, 203 (2008).

[36] E. Antillon, B. Wehefritz-Kaufmann, and S. Kais, Phys. Rev. E 85, 036706 (2012). 
[37] N. L. Guevara and A. V. Turbiner, Phys. Rev. A 84, 064501 (2011).

[38] M. Abramowitz, I. y Stegun (eds.), Handbook of mathematical functions, Dover Publications, 9 ed. New York, 1972.

[39] C. Froese Fischer, 2007,Advances In Atomic, Molecular, and Optical Physics vol. 55 eds. E. Arimondo, P.R. Berman, C.C. Lin. p 235. 